\title{
Analyse der Inhalte erziehungswissenschaftlicher Studiengänge im Hinblick auf eine spätere Tätigkeit in der berufsbezogenen Weiterbildung
}

\author{
Susanne Wißhak $\cdot$ Sabine Hochholdinger
}

Eingegangen: 16. Februar 2016 / Angenommen: 10. Mai 2016 / Online publiziert: 10. Juni 2016

(C) The Author(s) 2016. This article is available at SpringerLink with Open Access

Zusammenfassung Über die Qualifikationen von Lehrenden in der berufsbezogenen Weiterbildung ist bisher wenig bekannt. Ein bedeutsamer Anteil besitzt einen erziehungswissenschaftlichen Studienabschluss, außerdem scheinen zukünftige Weiterbildungsverantwortliche zur Zielgruppe erziehungswissenschaftlicher Studiengänge mit der Studienrichtung Erwachsenenbildung/Weiterbildung zu gehören. Die Studie befasst sich mit der Frage, welche Inhalte in Modulhandbüchern dieser Studiengänge vorgesehen sind, und ob diese für eine lehrende Tätigkeit in der berufsbezogenen Weiterbildung qualifizieren. Dazu wurden die Modulhandbücher von 32 Studiengängen inhaltsanalytisch ausgewertet und 10 Studiengangsverantwortliche mittels halbstrukturierter Interviews befragt. Während sich die Inhalte in den Modulhandbüchern größtenteils auf Personal- und Weiterbildungsmanagement, das deutsche Bildungswesen sowie Geschichte, Grundbegriffe und Theorien der Erziehungswissenschaft und Erwachsenenbildung beziehen, scheint mikrodidaktisches Handlungswissen, welches sich auf die Durchführung von Lehrveranstaltungen bezieht, eher nachrangig zu sein. Die Studiengangsverantwortlichen bestätigten die Aussagekraft der Modulhandbücher in Bezug auf die Studieninhalte. Eine Vorbereitung auf eine lehrende Tätigkeit durch das Studium sahen sie nur bedingt.

Schlüsselwörter Trainer · Berufliche Weiterbildung · Erziehungswissenschaftliche Studiengänge $\cdot$ Curriculumanalyse $\cdot$ Inhaltsanalyse $\cdot$ Experteninterviews

\footnotetext{
S. Wißhak $(\varangle) \cdot$ S. Hochholdinger

Universität Konstanz, Konstanz, Deutschland

E-Mail: Susanne.Wisshak@uni-konstanz.de

S. Hochholdinger

E-Mail: Sabine.Hochholdinger@uni-konstanz.de
} 


\title{
Analysis of the contents of study programs in educational sciences with regard to working as a training professional
}

\begin{abstract}
Little is known about the qualifications of trainers in further vocational education. Some hold a degree in educational sciences. At the same time prospective trainers seem to be part of the target group of programs that specialize in adult education. The study focuses on which contents are intended in these programs and whether they could qualify for a later occupational activity as a training professional. For this purpose the course descriptions of 32 programs were analyzed and 10 responsible program managers were interviewed. While topics of the programs mainly cover human resource- and further education management, the German education system, history, basic concepts and theories of educational sciences and adult education, microdidactic practical knowledge, which relates to the implementation of courses, seems to be subordinate. The responsibles of the programs confirmed the informative value of the course descriptions concerning the contents of the programs. They only saw a limited preparation for the trainer-job through the programs.
\end{abstract}

Keywords Trainers · Further vocational education · Educational sciences · Curriculum analysis $\cdot$ Content analysis $\cdot$ Expert interviews

\section{Lehrende in der berufsbezogenen Weiterbildung}

Durch berufsbezogene Weiterbildung wollen Arbeitnehmerinnen und Arbeitnehmer ihre beruflichen Chancen verbessern und Betriebe wettbewerbsfähig bleiben. Deutsche Unternehmen gaben Schätzungen zufolge im Jahr 2013 33,5 Milliarden Euro für die Weiterbildung ihrer Beschäftigten aus (vgl. Seyda und Werner 2014). Empirische Studien belegen daneben den individuellen, organisationalen und gesellschaftlichen Ertrag berufsbezogener Weiterbildung (vgl. Aiguinis und Kraiger 2009).

Berufsbezogene Weiterbildungsveranstaltungen werden in der internationalen Forschung meist als Trainings bezeichnet. Darunter sind ,,alle geplanten Maßnahmen zur Wiederherstellung, Erhaltung oder Erweiterung der beruflichen Handlungskompetenz" zu verstehen (Blickle 2014, S. 293). Auch deutschsprachige Lehrende in diesem Bereich bezeichnen sich selbst vornehmlich als „Trainer“ (Fuchs 2011, S. 169). Sie sind überwiegend freiberuflich tätig und werden von Unternehmen für die Planung und Durchführung von Fort- und Weiterbildungen (z. B. Führungstrainings, Verkaufstrainings, Schulungen in Projektmanagement) engagiert (Fuchs 2011). Damit sind sie nicht zu verwechseln mit betrieblichen Ausbildern.

Durch ihr professionelles Handlungswissen beeinflussen Trainerinnen und Trainer die Reaktionen und Lernergebnisse der Teilnehmenden (vgl. Sitzmann et al. 2008; Harris et al. 2014a, 2014b) ebenso wie den Transfererfolg (vgl. Burke und Hutchins 2008; Donovan und Darcy 2011; Harris et al. 2014a). Bedenkt man, dass es somit von diesen Personen abhängt, ob sich die Investitionen in Weiterbildung auszahlen, so verwundert es, dass über die Qualifikationen dieser Berufsgruppe bisher kaum etwas bekannt ist. Die Qualifizierung für den berufsbezogenen wie auch für den allgemeinen Weiterbildungsbereich ist in Deutschland nicht einheitlich geregelt. Das 
Deutsche Institut für Erwachsenenbildung (DIE) bemüht sich zwar seit Jahren um die Etablierung einheitlicher Standards; der von Kraft und Kollegen vorgeschlagene „Qualifikationsrahmen für die Weiterbildung“ wurde jedoch bisher nicht umgesetzt (Kraft et al. 2009, S. 61 ff.; Kraft 2013). Auch die Forschung liefert bisher nur wenige Erkenntnisse. Vereinzelte Befunde deuten darauf hin, dass sowohl in der allgemeinen wie auch in der berufsbezogenen Weiterbildung Tätige häufig einen pädagogischen bzw. erziehungswissenschaftlichen Studienabschluss, insbesondere mit der Studienrichtung Erwachsenen-/Weiterbildung (EB/WB) besitzen (Kraft 2006; Lenk 2010; Bonnes und Hochholdinger 2016). Wir stellten uns daher die Frage, welche Inhalte dort vorgesehen sind und inwiefern diese zur Qualifikation von Lehrenden in der berufsbezogenen Weiterbildung beitragen können.

\section{Forschungslage und Fragestellungen}

\subsection{Tätigkeitscharakteristika in der berufsbezogenen Weiterbildung}

Bisher gibt es weder ein umfassendes Anforderungsprofil für Lehrende in der berufsbezogenen Weiterbildung noch existiert ein eigenes Kompetenzmodell für diese Personengruppe. Jedoch lassen sich aus Befunden der Erwachsenenbildungsforschung, der internationalen Trainingsforschung und der empirischen Unterrichtsforschung teilweise deskriptiv, teilweise normativ, wesentliche Kernpunkte der Trainertätigkeit ableiten. Hierzu wurden Befragungen von Lehrenden und Teilnehmenden vorgenommen und insbesondere in der arbeitspsychologischen Wirksamkeitsforschung zahlreiche Studien zu Erfolgsfaktoren von Trainings durchgeführt. Demzufolge stellt zunächst die Lehrtätigkeit den Hauptbestandteil der berufsbezogenen Weiterbildung dar. Trainerinnen und Trainer planen und gestalten eigenständig ihre Veranstaltungen und führen sie selbst durch (vgl. Hippel und Fuchs 2009; Fuchs 2011; Kraft 2011; Schrader 2013; Alfänger et al. 2014; Marx et al. 2014a). Für diese Lehrtätigkeit sind der Literatur zufolge verschiedene Aktivitäten und Kompetenzfacetten relevant:

- Planung und Strukturierung von Lehrveranstaltungen, von der Bedarfsanalyse (vgl. Gauld und Miller 2004; Khamarko et al. 2012; Salas et al. 2012) bis zur Konzeption (Hippel und Fuchs 2009; Fuchs 2011).

- Einsatz und Variation verschiedener Lehr-Lernmethoden (vgl. Gauld und Miller, 2004; Burke und Hutchins 2008; Salas et al. 2012; Marx et al. 2014b).

- (lern-)psychologische Kenntnisse (z. B. zu Konzepten von Lernen und Transfer, Motivation und Emotionen sowie Befunde der Arbeitspsychologie) (vgl. Hutchins und Burke 2007; Salas et al. 2012; Marx et al. 2014b).

- Teilnehmerorientierung der didaktischen Gestaltung (vgl. Boendermaker et al. 2000, 2003; Hutchins 2009; Brotherton und Evans 2010; Khamarko et al. 2012).

- Umgang mit Gruppen, Kommunikation, Gestalten von Beziehungen und Konfliktmanagement (vgl. Boendermaker et al. 2000, 2003; Towler und Dipboye 2001; Gauld und Miller 2004; Terry 2010; Ghosh et al. 2012; Khamarko et al. 2012; Rasli et al. 2012; Marx et al. 2014b). In der Erwachsenenbildungsforschung bisher eher unüblich, in der empirischen Unterrichtsforschung jedoch sehr pro- 
minent ist in diesem Zusammenhang der Begriff des classroom management bzw. der „Klassenführung“ (Voss et al. 2015, S. 194).

- Trainingsevaluation wird teilweise genannt (vgl. Gauld und Miller 2004; Fuchs 2011), allerdings aus subjektiver Sicht mit geringerer Priorität als die objektiven Befunde der arbeits- und organisationspsychologischen Wirksamkeitsforschung nahelegen (z. B. Salas et al. 2012).

- Fachwissen über die Trainingsinhalte; auch eigene Praxiserfahrung wird häufig als wünschenswert angegeben (vgl. Russ-Eft et al. 2005; Hutchins 2009; Ghosh et al. 2012; Rasli et al. 2012).

Neben der Lehrtätigkeit werden insbesondere bei freiberuflichen Trainerinnen und Trainern unternehmerische und marketingbezogene Aktivitäten und Kenntnisse hervorgehoben (vgl. Fuchs 2011). Außerdem wird teilweise erwartet, dass Lehrende die Teilnehmenden über die Veranstaltungen hinaus beraten und begleiten (vgl. RussEft et al. 2005; Fuchs 2011; Khamarko et al. 2012).

Nachdem versucht wurde, ein grobes Anforderungsraster für berufsbezogenes Weiterbildungspersonal zu skizzieren, wird im Folgenden auf dessen Qualifizierung eingegangen.

\subsection{Erziehungswissenschaftliche Studienabschlüsse bei Lehrenden in der Weiterbildung}

Empirisch ist über die Bildungsbiografien von Trainerinnen und Trainern wenig bekannt. Die in Tab. 1 zusammengefassten Studien enthalten jedoch Hinweise darauf, dass Lehrende in der Weiterbildung häufig einen pädagogischen Studienabschluss besitzen, bzw. dass erziehungswissenschaftliche Studiengänge auf eine derartige Tätigkeit abzielen.

Schütz und Nittel (2012, S. 234) konstatieren eine „Dominanz pädagogischer Studiengänge in den Ausbildungsbiographien von Erwachsenenbildner/innen/ $\mathrm{n}$ “ mit $31 \%$. Andere Untersuchungen berichten einen Anteil pädagogischer Studienabschlüsse bei Weiterbildungspersonal zwischen $19 \%$ (WSF 2005; Lehramt ausgenommen) und $29 \%$ (Kraft 2006). Lenk (2010) untersuchte freiberufliches Weiterbildungspersonal und fand $14 \%$ mit einem erziehungswissenschaftlichen Studienabschluss, während in einer Interviewstudie von Bonnes und Hochholdinger (2016) ein Drittel der betrieblich Weiterbildenden angab, einen pädagogischen oder psychologischen Studienschwerpunkt gehabt zu haben. Speziell weiterführende Studiengänge zu EB/WB zielen zu 47,8 \% auf zukünftige Mitarbeitende in der Erwachsenen- und Weiterbildung ab (Faulstich und Graeßner 2003), und Ludwigsburger Studierende der Erziehungswissenschaft mit Schwerpunkt Erwachsenenbildung geben zu jeweils $40 \%$ den Berufswunsch „,betriebliche Aus- und Weiterbildung“ und „Personal- und Organisationsentwicklung“ an (Schüßler 2012, S. 135). Dem Diplom-PädagogenSurvey von Krüger et al. (2003) zufolge arbeiteten im Jahr 2001 32\% der Absolventinnen und Absolventen der Studienrichtung Erwachsenen- und Weiterbildung in einem entsprechenden Tätigkeitsfeld.

Obwohl die Forschung zum Weiterbildungspersonal einige Hinweise darauf gibt, dass Personen, welche in der allgemeinen und teilweise auch in der berufsbezoge- 


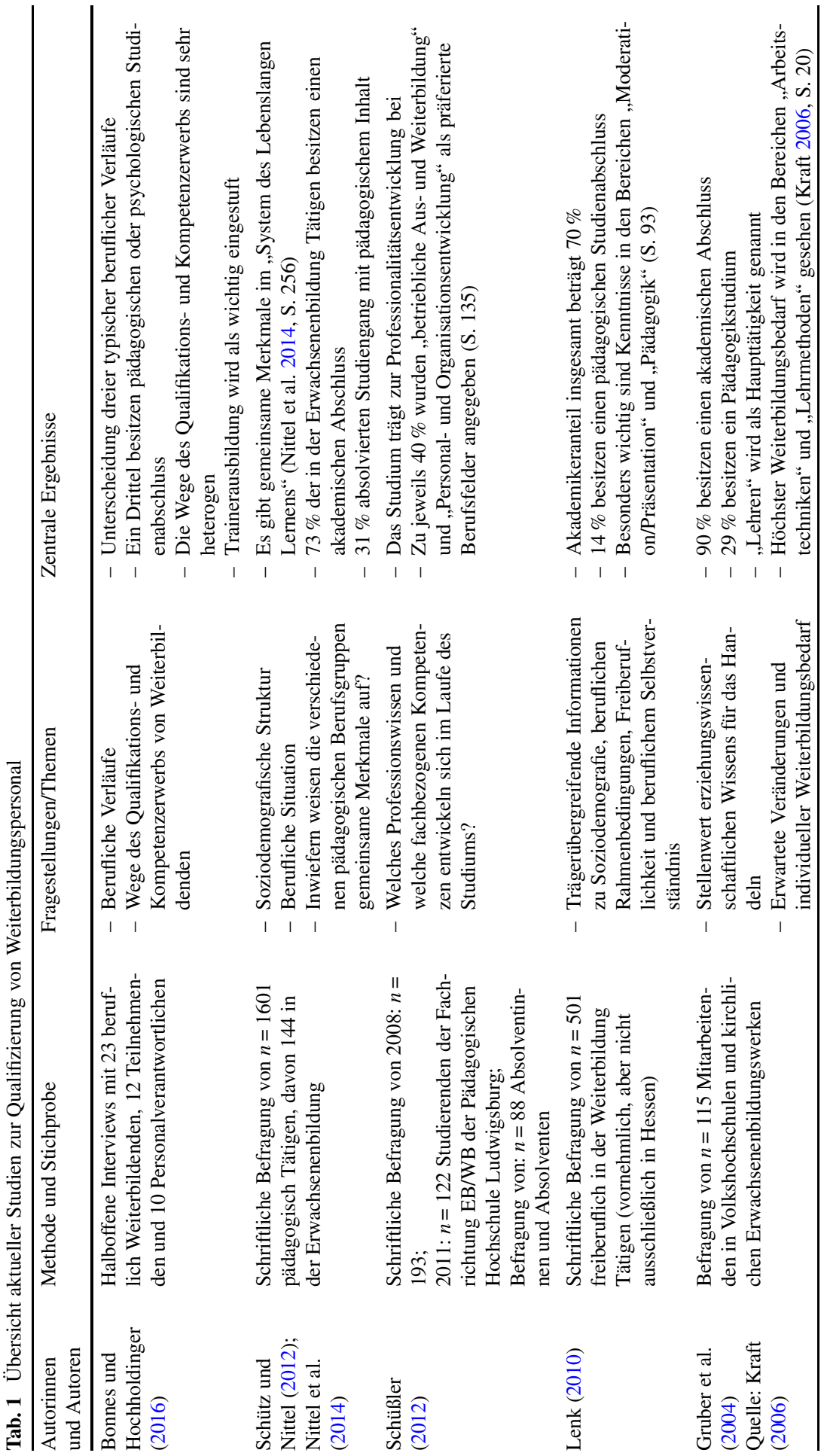




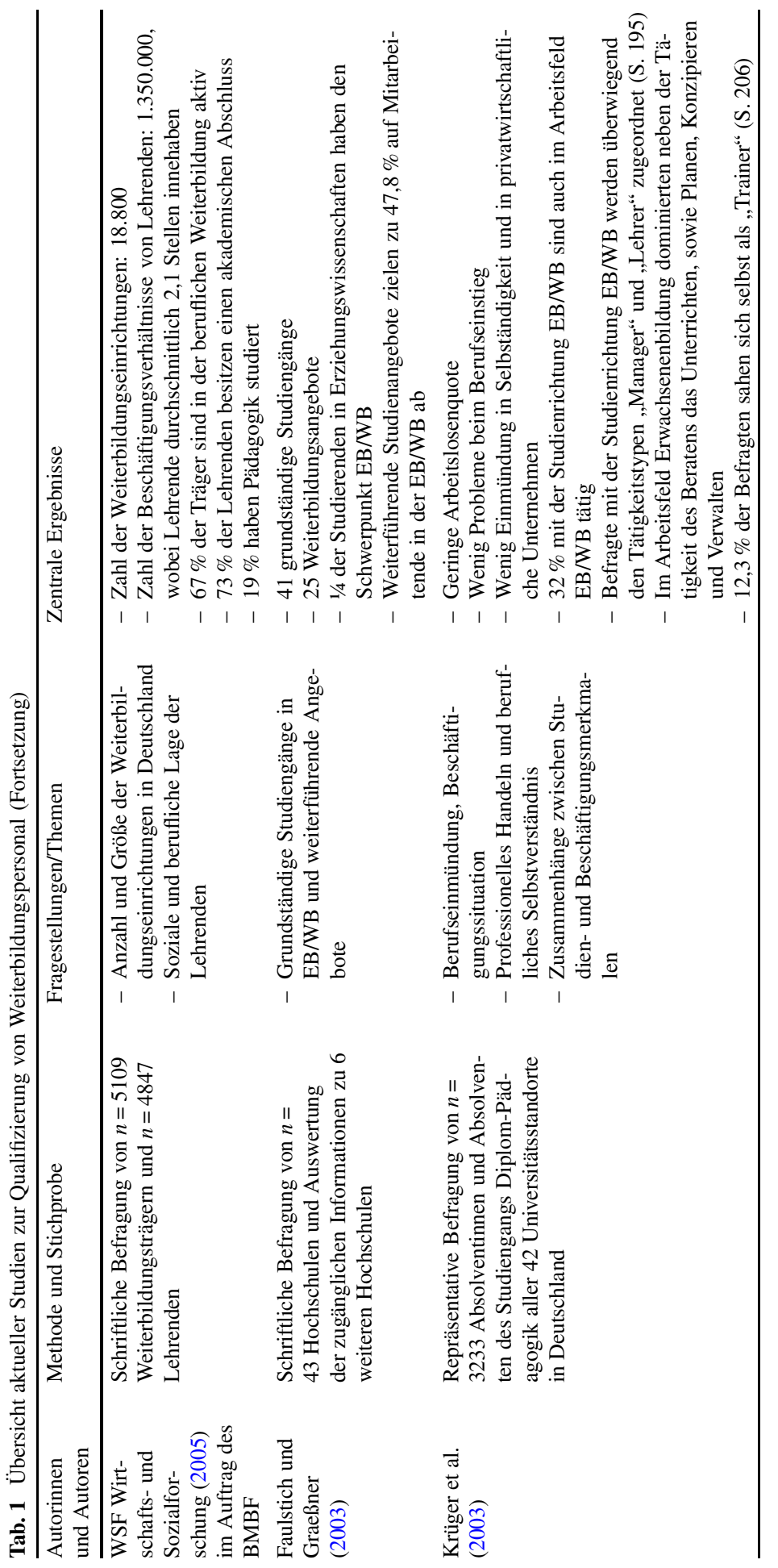


nen Weiterbildung tätig sind, einen erziehungswissenschaftlichen Studienabschluss besitzen, ist nicht klar, ob diese Studiengänge überhaupt darauf abzielen, zukünftige Trainerinnen und Trainer auszubilden.

Dieser Beitrag widmet sich daher erziehungswissenschaftlichen Studiengängen als einem potenziellen Qualifizierungsweg für Trainerinnen und Trainer. Es wurde untersucht, welche Studieninhalte durch die Modulhandbücher mit welcher Häufigkeit vorgegeben werden. Außerdem wurden Studiengangsverantwortliche gefragt, ob die Studiengänge aus ihrer Sicht auf eine spätere Trainertätigkeit vorbereiten, oder ob weitere Qualifikationen, wie etwa eine sogenannte Trainerausbildung, vonnöten sind.

Die Ergebnisse sollen mit den Tätigkeitscharakteristika berufsbezogenen Weiterbildungspersonals verglichen werden, um die Eignung erziehungswissenschaftlicher Studien als Vorbereitung für eine Trainertätigkeit zu diskutieren.

\section{Untersuchungsgegenstand}

Die Profile erziehungswissenschaftlicher Studiengänge zeichnen sich durch eine große Vielfalt aus. Anhand des Datenreport Erziehungswissenschaft (Thole et al. 2012), des Online-Studienführers des DIE und weiterer Internetportale wurden im Wintersemester 2013/14 insgesamt 111 Studiengänge an deutschen Hochschulen identifiziert, welche den Namen Erziehungswissenschaft, Pädagogik, Bildungswissenschaft, Erwachsenenbildung, Weiterbildung oder vergleichbare Titel trugen. Es wurden sowohl grundständige als auch weiterbildende Angebote erfasst. Studiengänge der Sozialpädagogik, der Wirtschaftspädagogik oder Lehramtsstudiengänge wurden nicht berücksichtigt. Von den 111 Studiengängen zielen 58 nach eigenen Angaben auf eine Tätigkeit

- in der beruflichen/betrieblichen Fort-/Weiterbildung und/oder

- in der Organisations- und/oder Personalentwicklung und/oder

- als Trainerin bzw. Trainer ab.

Diese fanden Berücksichtigung in den folgenden beiden Teilstudien, da die genannten Bereiche für eine spätere Trainertätigkeit einschlägig sind (vgl. Fuchs 2011). Es handelt sich um 26 Bachelor- und 32 Masterstudiengänge.

\section{Erste Teilstudie: Curriculumanalyse von 32 Modulhandbüchern}

\subsection{Methode}

Aus den 58 Studiengängen wurde eine nach Bachelor- und Masterstudiengängen geschichtete Stichprobe von 32 Studiengängen (55\%) gezogen und deren Modulhandbücher mithilfe der qualitativen Inhaltsanalyse nach Mayring (2010) ausgewertet. Die Stichprobe enthielt 15 Bachelor- und 17 Masterstudiengänge und ist vom Umfang her mit anderen Curriculumanalysen vergleichbar (vgl. Krattenmacher et al. 2010; Lohmann et al. 2011; Bauer et al. 2012; Hohenstein et al. 2014). Waren die 
jeweiligen Modulhandbücher nicht zugänglich, wurde auf Studien- oder Prüfungsordnungen zurückgegriffen. Die analysierten Dokumente umfassen insgesamt 1056 Seiten.

Da es sich um eine explorative Studie handelt, wurde das Kategoriensystem für die Inhaltsanalyse vornehmlich induktiv entwickelt, wobei jedoch die bestehende Literatur über Tätigkeitscharakteristika von Weiterbildungspersonal (vgl. Abschnitt 2.1) und zu pädagogischem Wissen von Lehrkräften (für einen Überblick vgl. Voss et al. 2015) berücksichtigt wurde. Von der Nutzung eines bereits existierenden Kategoriensystems, etwa des Kerncurriculums für konsekutive Bachelor-/Master-Studiengänge im Hauptfach Erziehungswissenschaft mit der Studienrichtung EB/WB der Deutschen Gesellschaft für Erziehungswissenschaft (Vorstand der DGfE 2010) wurde abgesehen, da dieses lediglich grobe Kategorien und ein Minimum an unverzichtbarem Grundlagenwissen enthält. Die sehr heterogene erziehungswissenschaftliche Studienlandschaft (vgl. Mania und Strauch 2010; Stisser et al. 2012) legt nahe, dass die Curricula der Universitäten davon abweichen, und dass durch ein ausschließlich deduktives Kategoriensystem nicht alle Inhalte erfasst werden können (vgl. Krattenmacher et al. 2010).

Das Selektionskriterium für die Codiereinheiten umfasst alle (Lern-)Inhalte, Kompetenzen, Lernergebnisse und Qualifikationsziele der Studiengänge, die in den Modulhandbüchern aufgeführt sind. Die Kategorien wurden mit Definitionen und Ankerbeispielen versehen, und es wurde ein Codierleitfaden erstellt. Die Codierung wurde von drei Personen mit der Software MAXQDA vorgenommen, wobei zunächst eine Codiererschulung erfolgte. Die geschichtete Zufallsstichprobe zur Ermittlung der Intercoderreliabilität umfasste vier Texte, was $13 \%$ des Materials entspricht. Der Intercoderreliabilitätskoeffizient nach Holsti (1969) beträgt .63. Kategorien, bei welchen systematische Fehler auftraten, wurden überarbeitet.

\subsection{Ergebnisse der Curriculumanalyse}

Das Kategoriensystem ist in acht Hauptkategorien gegliedert, welche 28 Unterkategorien umfassen (Tab. 2).

Insgesamt wurden 4572 Codierungen vorgenommen. Abb. 1 zeigt, dass 1493 Inhalte der Hauptkategorie A „Erziehungswissenschaftliche Grundlagen“ zugeordnet wurden. Ebenfalls sehr häufig vergeben wurde außerdem die Hauptkategorie B „Pädagogische Berufsfelder" mit 1406 Nennungen. Am dritthäufigsten fanden wir Codiereinheiten, die der Hauptkategorie D „Forschung “ zugeordnet werden konnten (580 Codierungen). Inhalte, welche sich explizit auf Lernen und Lernende beziehen, wurden 370 mal gefunden. Nach der „Planung und Gestaltung von Lehrveranstaltungen“ mit 330 Nennungen folgen die „Nachbardisziplinen“ der Erziehungswissenschaft (176 Codierungen) und der „Umgang mit Gruppen“ (147 Codierungen). Am wenigsten Inhalte wurden zur „Lernerfolgsmessung, Reflexion und Evaluation“ gefunden (70 Codierungen).

Häufigste Unterkategorie ist das „Personal- \& Weiterbildungsmanagement“ mit 792 Nennungen. Der Unterkategorie „Geschichte, Grundbegriffe und Theorien der Erziehungswissenschaft \& Erwachsenenbildung“ wurden 582 Codiereinheiten zugeordnet. Wie die Kategorie „Deutsches Bildungswesen“ mit 466 Codierungen gehört 
Tab. 2 Kategoriensystem mit Codierhäufigkeiten für Modulhandbücher von 32 Studiengängen

\begin{tabular}{|c|c|c|c|}
\hline \multirow[t]{2}{*}{$\begin{array}{l}\text { Haupt- } \\
\text { kategorie }\end{array}$} & \multirow[t]{2}{*}{$\begin{array}{l}\text { Unter- } \\
\text { kategorie }\end{array}$} & Bezeichnung der Kategorie & \multirow{2}{*}{$\begin{array}{l}\text { Anzahl } \\
\text { Codierungen } \\
4572\end{array}$} \\
\hline & & Gesamt & \\
\hline \multirow[t]{6}{*}{$\mathbf{A}$} & & Erziehungswissenschaftliche Grundlagen & 0 \\
\hline & 1 & $\begin{array}{l}\text { Geschichte, Grundbegriffe \& Theorien der } \\
\text { Erziehungswissenschaft \& Erwachsenenbil- } \\
\text { dung }\end{array}$ & 582 \\
\hline & 2 & Deutsches Bildungswesen & 466 \\
\hline & 3 & Gesellschaftliche Rahmenbedingungen & 156 \\
\hline & 4 & $\begin{array}{l}\text { Auseinandersetzung mit } \\
\text { Forschungsergebnissen }\end{array}$ & 256 \\
\hline & 5 & Theorie-Praxis-Bezug & 33 \\
\hline \multirow[t]{7}{*}{ B } & & Pädagogische Berufsfelder & 0 \\
\hline & 6 & Personal- \& Weiterbildungsmanagement & 792 \\
\hline & 7 & Organisationsentwicklung & 210 \\
\hline & 8 & Beratung & 111 \\
\hline & 9 & Soziale Arbeit \& Jugendarbeit & 224 \\
\hline & 10 & Schulorganisation & 46 \\
\hline & 11 & Frühpädagogik & 23 \\
\hline \multirow[t]{5}{*}{$\mathbf{C}$} & & Nachbardisziplinen & 8 \\
\hline & 12 & Psychologie & 90 \\
\hline & 13 & Soziologie/Sozialwissenschaften & 66 \\
\hline & 14 & Politikwissenschaft & 7 \\
\hline & 15 & Philosophie & 5 \\
\hline \multirow[t]{3}{*}{$\mathbf{D}$} & & Forschung & 0 \\
\hline & 16 & Wissenschaftstheorie & 124 \\
\hline & 17 & $\begin{array}{l}\text { Forschungsprojekte durchführen } \\
\text { (Methoden, Analyse) }\end{array}$ & 456 \\
\hline \multirow[t]{4}{*}{$\mathbf{E}$} & & Lernen \& Lernende & 0 \\
\hline & 18 & Grundlagen des Lernens & 224 \\
\hline & 19 & Didaktische Modelle & 115 \\
\hline & 20 & Umgang mit Heterogenität & 31 \\
\hline \multirow[t]{5}{*}{$\mathbf{F}$} & & $\begin{array}{l}\text { Planung \& Gestaltung von Lehrveranstal- } \\
\text { tungen }\end{array}$ & 40 \\
\hline & 21 & Bedarfsanalyse \& Lernziele & 69 \\
\hline & 22 & Lernumgebung \& Ablauf planen & 101 \\
\hline & 23 & Lehr-/Lernmethoden, Medien \& Lernmaterial & 115 \\
\hline & 24 & Transferorientierung & 5 \\
\hline \multirow[t]{5}{*}{$\mathbf{G}$} & & Umgang mit Gruppen & 0 \\
\hline & 25 & Gruppenprozesse verstehen und begleiten & 49 \\
\hline & 26 & Kommunikationsfähigkeit & 44 \\
\hline & 27 & Führung & 29 \\
\hline & 28 & Konfliktmanagement & 25 \\
\hline $\mathbf{H}$ & & $\begin{array}{l}\text { Lernerfolgsmessung, } \\
\text { Reflexion \& Evaluation }\end{array}$ & 70 \\
\hline
\end{tabular}




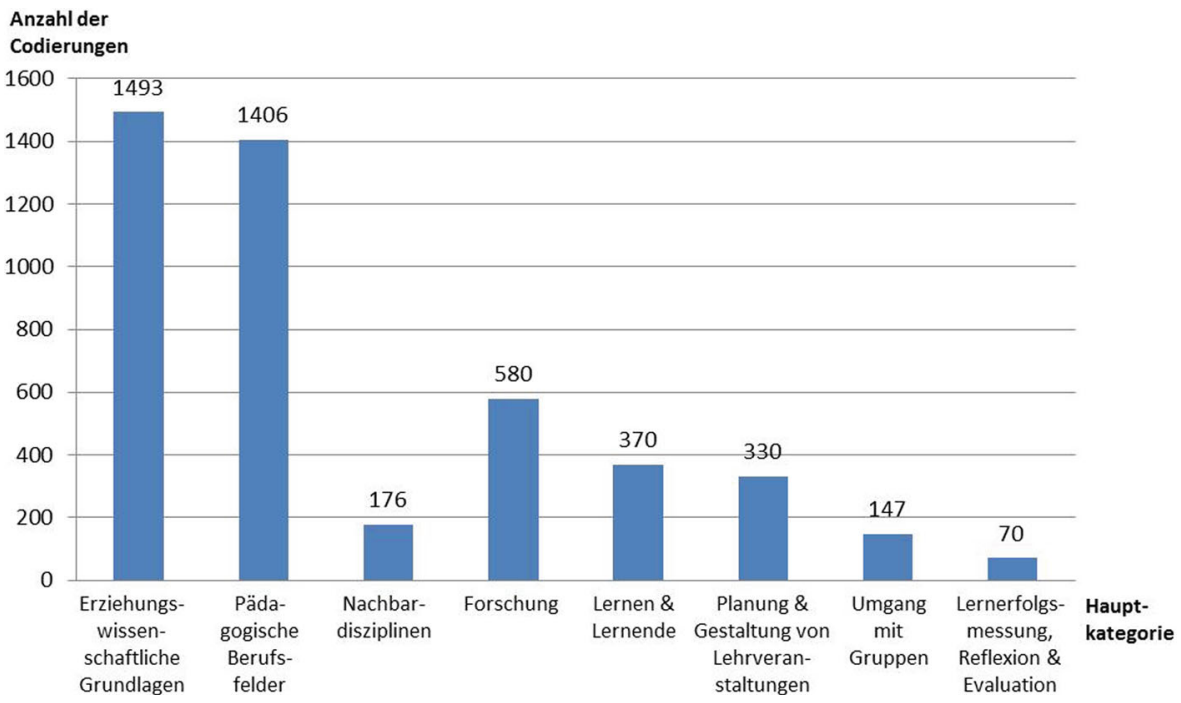

Abb. 1 Verteilung der Codierungen in 32 Modulhandbüchern auf die Hauptkategorien

—Prozentuale Häufigkeit in den Bachelorstudiengängen

■ Prozentuale Häufigkeit in den Masterstudiengängen

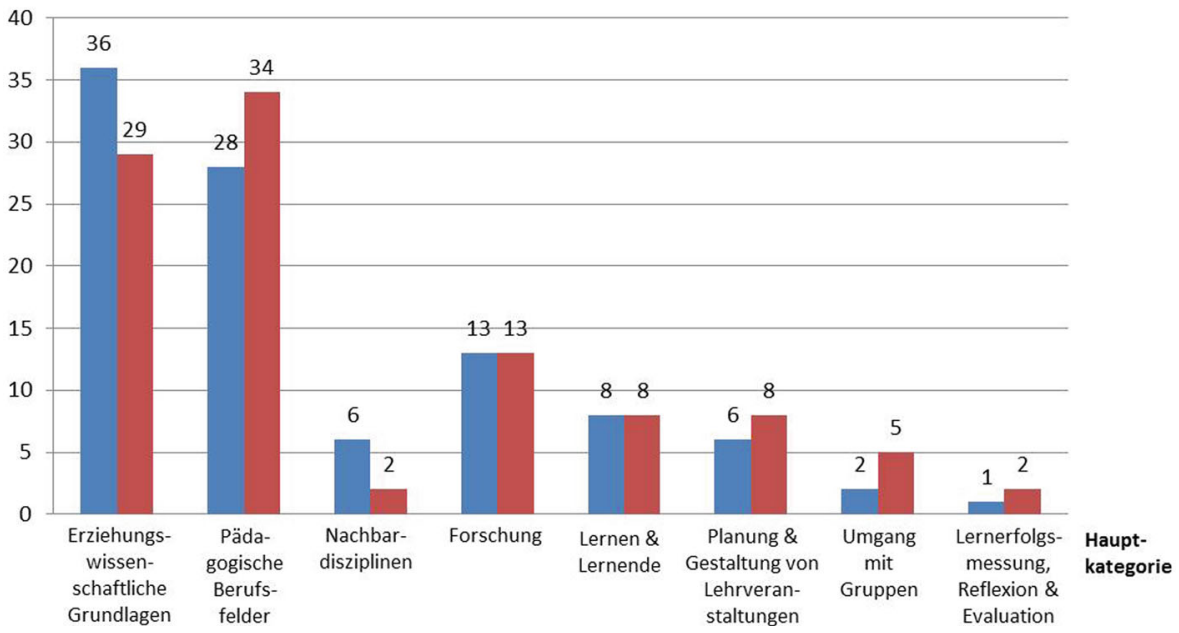

Abb. 2 Prozentuale Verteilung der Codierungen auf die Hauptkategorien getrennt nach Bachelor- und Masterstudiengängen (Prozentwerte gerundet)

sie den „Erziehungswissenschaftlichen Grundlagen“ an. Außerdem ist die Unterkategorie „Forschungsprojekte durchführen“ mit 456 Codierungen stark vertreten. Diese Kategorie beinhaltet bspw. Erhebungsmethoden und Analyseverfahren.

Abb. 2 zeigt die Codierhäufigkeiten der Hauptkategorien nach Bachelor- und Masterstudiengängen getrennt. Der Vergleich macht deutlich, dass in den Bachelorstudiengängen mehr erziehungswissenschaftliche Grundlagen vorgesehen sind und 
mehr Lernangebote in den Nachbardisziplinen bestehen. In den Masterstudiengängen sind mehr Inhalte zu pädagogischen Berufsfeldern vorgesehen, vor allem zum Personal- und Weiterbildungsmanagement. Außerdem gibt es bei den Themen „Planung \& Gestaltung von Lehrveranstaltungen“, „Umgang mit Gruppen“ und „Lernerfolgsmessung, Reflexion \& Evaluation“ einen deutlichen Unterschied zugunsten der Masterstudiengänge.

\section{Zweite Teilstudie: Leitfadengestützte Experteninterviews}

\subsection{Methode}

In einem weiteren Schritt befragten wir zwei Professorinnen und vier Professoren sowie vier Mitarbeitende ( 2 weiblich, 2 männlich) an erziehungswissenschaftlichen Lehrstühlen mittels leitfadengestützter Interviews. Die Befragten stellen eine Gelegenheitsstichprobe von Studiengangsverantwortlichen aus der Gruppe der 58 einschlägigen Studiengänge dar. Der Interviewleitfaden wurde zunächst in drei Interviews getestet und optimiert. Die Haupterhebung in Form zehn telefonischer Interviews fand von Juni bis August 2014 statt.

Die Befragten waren durchschnittlich seit drei Jahren und neun Monaten für den betreffenden Studiengang zuständig, und sieben der zehn Expertinnen und Experten waren an Aufbau oder Weiterentwicklung der Studiengänge beteiligt. Vier der Befragten konnten über jeweils zwei Studiengänge Auskunft geben, so dass Informationen über 14 Studiengänge (5 Bachelor, 9 Master) vorliegen. Der Interviewleitfaden enthielt folgende Leitfragen:

1. Wie weit bereitet der Studiengang auf eine spätere Trainertätigkeit vor?

2. Beinhaltet das Modulhandbuch die tatsächlichen Inhalte des Studiengangs?

3. Sind Absolventinnen und Absolventen des Studiengangs als Trainerin oder Trainer tätig, und besitzen diese eine zusätzliche Trainerausbildung?

4. Halten Sie eine verpflichtende Trainerausbildung als Praxisphase nach dem Pädagogikstudium für angehende Trainerinnen und Trainer für realisierbar?

Für die Auswertung der Interviews wurde abermals die qualitative Inhaltsanalyse nach Mayring (2010) herangezogen. Anhand der Fragestellungen wurden zunächst deduktive Kategorien gebildet und eine skalierende Strukturierung vorgenommen. Tab. 3 stellt beispielhaft die Kategorie ,,Vorbereitung des Studiengangs auf die Trainertätigkeit" dar. Außerdem wurden in einem weiteren Schritt induktive Kategorien gebildet, welche gemeinsam mit den Ergebnissen der skalierenden Analyse in Abschn. 5.2 vorgestellt werden.

\subsection{Ergebnisse der Experteninterviews}

Lediglich vier der 14 Studiengänge bereiten nach Angabe der Befragten ausreichend auf eine Tätigkeit als berufsbezogenes Weiterbildungspersonal vor. Drei davon befassen sich dem Titel nach explizit mit Erwachsenenbildung. Am häufigsten lassen sich die Antworten der Kategorie ,Vorbereitung findet teilweise statt“ zuordnen. Oft 
Tab. 3 Ausprägungen der Kategorie „Vorbereitung des Studiengangs auf die Trainertätigkeit“

\begin{tabular}{|c|c|c|c|}
\hline Ausprägung & Kategoriendefinition & Ankerbeispiel & Codierregeln \\
\hline $\begin{array}{l}\text { Vorbereitung } \\
\text { findet statt }\end{array}$ & $\begin{array}{l}\text { Studierende werden } \\
\text { durch das Studium } \\
\text { ausreichend auf eine } \\
\text { Trainertätigkeit in } \\
\text { der berufsbezoge- } \\
\text { nen Weiterbildung } \\
\text { vorbereitet }\end{array}$ & $\begin{array}{l}\text { „Einmal zu den Tätigkeiten Kon- } \\
\text { zeption, Durchführung, Evaluation } \\
\text { haben wir einmal theoretische } \\
\text { Studieninhalte [...], die sich mit } \\
\text { Didaktik, Makro- sowie auch } \\
\text { Mikrodidaktik von Kursplanung } \\
\text { beschäftigen. Zur Durchführung } \\
\text { methodisch und Medieneinsatz } \\
\text { auch thematisieren ... Evaluati- } \\
\text { on ist auch ein Modul unseres } \\
\text { Studiengangs, sodass das auch } \\
\text { abgedeckt ist von der inhaltlichen } \\
\text { Art“ (GP 5, Z. 72ff) }\end{array}$ & $\begin{array}{l}\text { Inhalte des Studiums } \\
\text { befähigen ausreichend } \\
\text { zu einer Trainertätig- } \\
\text { keit im Anschluss an } \\
\text { das Studium. } \\
\text { Nennung zahlreicher } \\
\text { relevanter Inhalte. } \\
\text { Die Antwort lautet „Ja“ } \\
\text { ohne Einschränkungen }\end{array}$ \\
\hline $\begin{array}{l}\text { Vorbereitung } \\
\text { findet } \\
\text { teilweise } \\
\text { statt }\end{array}$ & $\begin{array}{l}\text { Studierende werden } \\
\text { durch das Studium } \\
\text { teilweise auf eine } \\
\text { Trainertätigkeit in } \\
\text { der berufsbezoge- } \\
\text { nen Weiterbildung } \\
\text { vorbereitet }\end{array}$ & $\begin{array}{l}\text { „[...] dass da dann natürlich schon } \\
\text { Grundlagen gelegt werden für eine } \\
\text { Trainertätigkeit [...]“(GP } 7, \mathrm{Z} \text {. } \\
80 \text { f) }\end{array}$ & $\begin{array}{l}\text { Studium bereitet teil- } \\
\text { weise auf spätere Trai- } \\
\text { nertätigkeit vor. } \\
\text { Es werden Grundlagen } \\
\text { geschaffen. } \\
\text { Es fehlen aber auch } \\
\text { Inhalte. } \\
\text { Studierende müssen } \\
\text { sich noch zusätzlich } \\
\text { Wissen oder Fertigkei- } \\
\text { ten aneignen }\end{array}$ \\
\hline $\begin{array}{l}\text { Vorbereitung } \\
\text { findet kaum } \\
\text { bis gar nicht } \\
\text { statt }\end{array}$ & $\begin{array}{l}\text { Studierende werden } \\
\text { nur in geringem Um- } \\
\text { fang bis gar nicht auf } \\
\text { eine Trainertätigkeit } \\
\text { vorbereitet }\end{array}$ & $\begin{array}{l}\text { „Der Bachelorabschluss bei uns } \\
\text { taugt für Ihre Idee nicht“ (GP } 3 \mathrm{Z} \text {. } \\
141 \mathrm{f} \text { ) }\end{array}$ & $\begin{array}{l}\text { Die wesentlichen In- } \\
\text { halte fehlen. } \\
\text { Es wird eher auf an- } \\
\text { dere Berufe oder An- } \\
\text { schlussstudiengänge } \\
\text { vorbereitet }\end{array}$ \\
\hline
\end{tabular}

werden „Grundlagen“ für eine Trainertätigkeit geschaffen (GP 1, Z. 78; GP 7, Z. 74, 81). Die Studierenden erlernen zwar u. a. Fertigkeiten, welche für den Trainerberuf nützlich sein können, dies ist häufig jedoch kein vorrangiges Ziel des Studiengangs:

Das heißt nicht, dass das nicht durchaus auch sein kann und dass sie das auch durchaus werden. Aber ich würde sagen, das ist nicht die grundlegende Zielrichtung (...) (GP 10, Z. 89 ff.).

Vier der Befragten merkten an, die Studiengänge zielten eher auf planende bzw. leitende Tätigkeiten ab, als auf eine direkte Lehrtätigkeit.

Wenn Sie sagen, dass Sie gerade auf der Interaktionsebene das verorten (...) Dann würde ich (...) sagen, das ist nicht der Schwerpunkt des Studiengangs. Also wir haben nicht die Ausbildungsperspektive für Personen, die jetzt in Lehr-Lerngeschehen, direkt in die Interaktion quasi gehen, (...) sondern eher in der Perspektive disponierend, planerisch tätig, organisierende Tätigkeiten (...) (GP 10, Z. 72 ff.). 
Tab. 4 Induktive Kategorien zur Frage, welche Inhalte in den Studiengängen fehlen, die für eine Trainertätigkeit wichtig wären

\begin{tabular}{ll}
\hline Bezeichnung der Kategorie & Anzahl Codierungen \\
Gesamt & 43 \\
\hline Umgang mit Gruppen & 12 \\
Lehrperson (z. B. Selbstmanagement) & 10 \\
Anwendungsorientierung/Praxisbezug & 8 \\
Planung \& Gestaltung von Lehrveranstaltungen & 8 \\
Unternehmerisches Wissen (Akquise, Abrechnung) & 2 \\
Erwachsenenbildung & 1 \\
Beratung & 1 \\
Teilnehmerorientierung & 1 \\
\hline
\end{tabular}

Fünf Studiengänge wurden als nicht zielführend für die Trainertätigkeit eingestuft. Die Inhalte, welche nach Meinung der Befragten in den Studiengängen fehlten, um auf eine Trainertätigkeit vorzubereiten, wurden gesammelt und zu Kategorien zusammengefasst (Tab. 4). Diese decken sich teils mit unserem Kategoriensystem, es ergaben sich jedoch auch neue Themen.

Eine Problematik, auf welche drei der Befragten hinwiesen, war, dass die betreffenden Studiengänge hohe Wahlanteile aufweisen und deshalb die besuchten Veranstaltungen stark von individuellen Schwerpunktsetzungen der Studierenden abhängen. Die Interviewten sprachen damit ein Problem an, von welchem bereits Lohmann et al. (2011) berichten.

Bezüglich der Aussagefähigkeit der Modulhandbücher gaben sieben der zehn Befragten an, dass die Modulhandbücher den Inhalten der Studiengänge entsprechen. Einschränkend wurde genannt, dass die Texte bei Änderungen nicht rechtzeitig aktualisiert werden, oder dass es Inhalte gibt, die nicht explizit in den Dokumenten ausformuliert sind. Lediglich eine Person stellte die Aussagekraft der Modulhandbücher grundsätzlich infrage.

Des Weiteren wollten wir wissen, ob die Absolventinnen und Absolventen der betreffenden Studiengänge später im Trainerberuf tätig sind. In einem der Masterstudiengänge stellt eine vorausgehende Tätigkeit in der Weiterbildung eine Zulassungsvoraussetzung dar; dementsprechend arbeiten auch viele Absolventinnen und Absolventen wieder in dem Bereich. Zwei Befragte konnten sagen, dass viele ihrer Alumni später im Trainerberuf arbeiten. Vier gaben an, die ehemaligen Studierenden fänden teilweise in den Trainerberuf, zwei schlossen dies aus.

Fünf der Befragten gaben an, dass ihre Studierenden vor, während oder nach dem Studium Zusatzqualifikationen erwerben. Genannt wurden der Ausbilderschein der IHK (,AEVO“), Coachingausbildungen oder „Lernbegleiter-Kurs[e]“ (GP 8, Z. 252 ff.). An einer Universität wird ein eigener Train-the-Trainer-Kurs angeboten.

Zuletzt fragten wir nach einer obligatorischen Praxisausbildung mit einheitlichen Qualitätsstandards, vergleichbar mit dem Vorbereitungsdienst für Lehrkräfte oder der Psychotherapieausbildung. Lediglich eine Person hielt dies für realisierbar. Es wurden vor allem die Dauer, Kosten und mangelnde Flexibilität sowie die Organisation und Zuständigkeiten als Schwierigkeiten gesehen. Sechs Personen sprachen sich 
jedoch für eine stärkere Verzahnung von Theorie und Praxis innerhalb des Studiums aus, bspw. durch Praktika, Praxissemester oder Mentoringprogramme.

\section{Diskussion}

\subsection{Zusammenfassung der Ergebnisse}

Die Studie erfasste mittels einer Analyse von Modulhandbüchern, welche Inhalte in 32 pädagogischen Studiengängen vorgesehen sind, und erhob mittels einer mündlichen Befragung von zehn Expertinnen und Experten, inwiefern diese auf eine spätere Trainertätigkeit vorbereiten.

In den Modulhandbüchern liegt ein Schwerpunkt auf der Geschichte, den Grundbegriffen und Theorien der Erziehungswissenschaft. Sie sehen vor, dass die Studierenden sich mit unserem und anderen Bildungssystemen auseinandersetzen und sich Wissen über Organisationsentwicklung und Weiterbildungsmanagement aneignen. Die interviewten Studiengangsverantwortlichen wiesen darauf hin, dass die Studierenden auf leitende bzw. planende Tätigkeiten in Bildungsorganisationen vorbereitet werden. Dabei geht es bspw. um strategische und konzeptionelle Tätigkeiten wie die Angebotsplanung. Laut Schütz und Nittel (2012) verfügen dementsprechend insbesondere leitende Angestellte in Bildungseinrichtungen über ein Pädagogikstudium. Auch der Bereich „Forschung“ ist in den Modulhandbüchern stark vertreten. In der Praxis zeigt sich jedoch eine Diskrepanz zwischen der ausführlichen Beschäftigung mit Forschungsmethoden im Studium und der geringen Anwendung im Beruf (vgl. Schüßler 2012).

Eine verbindliche Praxisphase nach dem Schema des Vorbereitungsdienstes von Lehrkräften sahen die in unserer Studie befragten Studiengangsverantwortlichen kritisch und bezweifelten vor allem die Umsetzbarkeit. Sie schlugen stattdessen eine stärkere Verknüpfung von Theorie und Praxis bereits während des Studiums vor, was auch in der Literatur empfohlen wird (vgl. ebd.). Eine Trainerausbildung nach dem Pädagogikstudium halten sie jedoch grundsätzlich für sinnvoll.

\subsection{Eingeschränkte Vorbereitung auf die Trainertätigkeit}

Gemäß dem Tätigkeitsprofil des Trainerberufs (Abschn. 2.1) stellt die Lehrtätigkeit den Kern des Trainerhandelns dar. Dazu gehört zunächst pädagogisches Planungsund Handlungswissen. Die Planung und Gestaltung von Lehrveranstaltungen betrifft jedoch lediglich 7,2\% der untersuchten Studieninhalte, wovon 2,5\% auf die LehrLernmethoden entfallen. Grundlagenwissen über Lernen und Lernende scheint etwas häufiger vorgesehen zu sein: Die entsprechende Kategorie enthält 8,1\% aller Codiereinheiten, und in den Nachbardisziplinen Psychologie und Soziologie beschäftigen sich die Studierenden außerdem mit Entwicklungs-, Motivations- und Emotionstheorien sowie mit Sozialisationsprozessen. Aspekte der Teilnehmerorientierung sind hingegen in den Modulhandbüchern kaum explizit zu finden, wobei Inhalte zur Erwachsenenbildung und zum Weiterbildungsmanagement diese wahrscheinlich implizit enthalten. Der Umgang mit Heterogenität scheint sehr vereinzelt 
thematisiert zu werden $(0,7 \%)$, was eine gewisse Sensibilisierung für verschiedene Teilnehmerbedürfnisse nahelegt. Auch Inhalte zum Umgang mit Gruppen (3,2\%) sind in den Modulhandbüchern kaum vertreten. Auf Erfolgsmessung und Evaluation wird, verglichen mit den anderen Hauptkategorien, am wenigsten Wert gelegt $(1,5 \%)$, wobei davon ausgegangen werden kann, dass die Studierenden die methodischen Kompetenzen dafür dennoch erwerben, da die Modulhandbücher eine ausführliche Beschäftigung mit Forschungsmethoden und statistischen Analyseverfahren vorsehen. Ein Aspekt, den die Studiengänge für die meisten Trainingsthemen nicht abdecken können, ist das Fachwissen. Dieser Teil professioneller Handlungskompetenz muss häufig auf anderem Wege erlangt werden. Unternehmerische Fähigkeiten erlangen die Studierenden ggf. in geringem Maße im Bereich Personalund Weiterbildungsmanagement, zu welchem bspw. auch Bildungsmarketing und -controlling zählen. Auf beratende Tätigkeiten außerhalb der Lehrveranstaltungen werden die Studierenden vermutlich ebenfalls in geringem Maße vorbereitet, da Beratung als potenzielles Berufsfeld von Pädagogikstudierenden teilweise adressiert wird $(2,4 \%)$.

Auf Basis der Ergebnisse lässt sich vermuten, dass erziehungswissenschaftliche Studiengänge nur bedingt auf eine Trainertätigkeit vorbereiten. Das gilt selbst für solche Studienprofile, welche nach eigenen Angaben auf Weiterbildung abzielen. Die Studierenden scheinen kaum auf mikrodidaktische Lehr-Lerngestaltung vorbereitet zu werden. Dies spiegelt auch die Absolventenbefragung von Schüßler (2012) wider. In Bezug auf Kompetenzen zur Administration und Durchführung von Kursen, Bedarfsanalyse, Umgang mit unterschiedlichen Adressaten und Umgang mit Gruppendynamik, gaben die von Schüßler Befragten an, dass sie in höherem Maße in der Erwerbstätigkeit gefordert werden als sie im Studium erlernt wurden. Schüßler geht außerdem davon aus, dass die Ausbildung einer erwachsenenbildnerischen Professionalität überhaupt erst im Masterstudium stattfindet. Dies können unsere Daten insofern bestätigen, als in den Modulhandbüchern der Masterstudiengänge mehr handlungs- und berufsbezogene Inhalte gefunden wurden als in denen der Bachelorstudiengänge.

Die Ergebnisse der Curriculumanalyse sind konsistent mit den Antworten der befragten Expertinnen und Experten. Das Studium scheint Grundlagen zu liefern bzw. - im Fall von weiterbildenden Masterstudiengängen - eine theoretische Verankerung der bereits vorhandenen Fähigkeiten darzustellen. Es enthält aber wenig prozedurales Wissen für eine lehrende Tätigkeit. Aus den Aussagen der Studiengangsverantwortlichen geht außerdem hervor, dass es auch kein vorrangiges Ziel der Studiengänge ist, zukünftige Trainerinnen und Trainer auszubilden. Dementsprechend machen viele Studierende zusätzlich eine Trainer- oder Coachingausbildung. Dies entspricht den Befunden von Fuchs (2011), denen zufolge Trainerinnen und Trainer ein erziehungswissenschaftliches Studium zwar als gute Grundlage einschätzten, gleichzeitig aber angaben, dass eine akademische Ausbildung keine hinreichende Qualifikation für erfolgreiches Trainerhandeln darstellt. Auch in der Studie von Bonnes und Hochholdinger (2016) wurde der Nutzen von Trainerausbildungen als hoch eingeschätzt, und die Analyse von Wißhak und Hochholdinger (2015) legt nahe, dass die Themen, welche in Trainerausbildungen behandelt werden, ein erziehungswissenschaftliches Studium gut ergänzen können. 


\subsection{Limitationen und Perspektiven}

Die Curriculumanalyse wird in der empirischen Bildungsforschung häufig zur Erfassung von Studieninhalten eingesetzt. Gleichzeitig wird auf Schwierigkeiten hingewiesen, die entstehen, wenn sich die Texte in ihrem Abstraktionsniveau stark unterscheiden (vgl. Hohenstein et al. 2014), was bei Modulhandbüchern häufig der Fall ist. Außerdem ist zu bedenken, dass Letztere lediglich die intendierten Inhalte von Studiengängen preisgeben (vgl. Lohmann et al. 2011). Wie weit Lehrveranstaltungen diese abdecken, und wie weit sie von den Studierenden erlernt und später umgesetzt werden, lässt sich aus der Inhaltsanalyse nicht schließen. Die befragten Studiengangsverantwortlichen konnten jedoch bestätigen, dass die jeweiligen Modulhandbücher die Inhalte ihrer Studiengänge abbilden.

Aus forschungsökonomischen Gründen wurde die Stichprobe auf 32 Studiengänge beschränkt. Sie ist dennoch umfangreicher als die meisten vergleichbaren Curriculumanalysen (vgl. Krattenmacher et al. 2010; Lohmann et al. 2011; Bauer et al. 2012; Hohenstein et al. 2014). Die überwiegend induktive Entwicklung des Kategoriensystems ist ein aufwendiges Verfahren, welches die Objektivität einer Untersuchung verringern kann. Ihr wurde angesichts des explorativen Charakters der Untersuchung der Vorzug gegeben.

Die Frage, welches Wissen Trainerinnen und Trainer brauchen, um ihren Beruf erfolgreich ausüben zu können, ist bisher nicht ausreichend geklärt. Trotz einzelner Tätigkeitsprofile und den Ergebnissen aus der arbeits- und organisationspsychologischen Wirksamkeitsforschung ist bislang nicht empirisch erfasst worden, welches Wissen tatsächlich relevant für Trainerhandeln ist. Deshalb ist weitere Forschung auf diesem Gebiet nötig.

Die Studie beleuchtet Inhalte erziehungswissenschaftlicher Studiengänge im Hinblick auf eine spätere Trainertätigkeit. Die Erforschung der Qualifizierungswege und des benötigten Wissens soll es ermöglichen, ihre Ausbildung langfristig zu optimieren. Die Untersuchung liefert Hinweise darauf, dass ein erziehungswissenschaftliches Studium zwar eine gute Basis, jedoch keine hinreichende Qualifizierung für eine lehrende Tätigkeit in der berufsbezogenen Weiterbildung darstellt.

Open Access Dieser Artikel wird unter der Creative Commons Namensnennung 4.0 International Lizenz (http://creativecommons.org/licenses/by/4.0/deed.de) veröffentlicht, welche die uneingeschränkte Nutzung, Verbreitung und Wiedergabe für beliebige Zwecke erlaubt, sofern Sie den/die ursprünglichen Autor(en) und die Quelle ordnungsgemäß nennen, einen Link zur Creative Commons Lizenz beifügen und angeben, ob Änderungen vorgenommen wurden.

\section{Literatur}

Aguinis, H., \& Kraiger, K. (2009). Benefits of training and development for individuals and teams, organizations, and society. Annual Review of Psychology, 60(1), 451-474.

Alfänger, J., Cywinski, R., \& Elias, A. (2014). Einkommensverhältnisse, Tätigkeiten und Selbstwahrnehmungen des Weiterbildungspersonals im Wandel - Ergebnisse einer Online-Befragung. In Deutsches Institut für Erwachsenenbildung (Hrsg.), DIE spezial. Trends der Weiterbildung. DIE-Trendanalyse 2014 (S. 69-79). Bielefeld: W. Bertelsmann.

Bauer, J., Diercks, U., Rösler, L., Möller, J., \& Prenzel, M. (2012). Lehramtsstudium in Deutschland: Wie groß ist die strukturelle Vielfalt? Unterrichtswissenschaft, 40(2), 101-120. 
Blickle, G. (2014). Personalentwicklung. In F. W. Nerdinger, G. Blickle, \& N. Schaper (Hrsg.), SpringerLehrbuch. Arbeits- und Organisationspsychologie (3. Aufl. S. 291-318). Berlin: Springer.

Boendermaker, P.M., Conradi, M.H., Schuling, J., Meybloom-de Jong, B., Zwierstra, R.P., \& Metz, J.C.M. (2003). Core characteristics of the competent general practice trainer, a Delphi study. Advances in Health Sciences Education, 8(2), 111-116.

Boendermaker, P. M., Schuling, J., Meybloom-de Jong, B., Zwierstra, R. P., \& Metz, J.C. M. (2000). What are the characteristics of the competent general practitioner trainer? Family Practice, 17(6), 547-553.

Bonnes, C., \& Hochholdinger, S. (2016). Beruf TrainerIn? Die berufliche Entwicklung und die Wege des Qualifikations- und Kompetenzerwerbs des Weiterbildungspersonals in der beruflichen und betrieblichen Weiterbildung.bwp@ (29. Update), http://www.bwpat.de/ausgabe29/bonnes_hochholdinger_ bwpat29.pdf. Zugegriffen: 2. Juni 2016

Brotherton, J., \& Evans, C. (2010). The importance of the trainer: factors affecting the retention of clients in the training services sector. Industrial and commercial training, 42(1), 23-31.

Burke, L. A., \& Hutchins, H. M. (2008). A study of best practices in training transfer and proposed model of transfer. Human Resource Development Quarterly, 19(2), 107-128.

DGfE - Vorstand der Deutschen Gesellschaft für Erziehungswissenschaft (Hrsg.). (2010). Kerncurriculum Erziehungswissenschaft. Empfehlungen der Deutschen Gesellschaft für Erziehungswissenschaft (DGfE). Erziehungswissenschaft, 21 (Sonderband).

Donovan, P., \& Darcy, D. P. (2011). Learning transfer: the views of practitioners in Ireland. International Journal of Training and. Development, 15(2), 121-139.

Faulstich, P., \& Graeßner, G. (2003). Studiengänge Weiterbildung in Deutschland. http://www.die-bonn. de/esprid/dokumente/doc-2003/faulstich03_03.pdf. Zugegriffen: 10. Mai 2016.

Fuchs, S. (2011). Professionalitätsentwicklung des Weiterbildungspersonals: Tätigkeiten, Kompetenzen und Fortbildung von Trainern in der beruflichen/betrieblichen Weiterbildung. Hamburg: Dr. Kovac.

Gauld, D., \& Miller, P. (2004). The qualifications and competencies held by effective workplace trainers. Journal of European Industrial. Training, 28(1), 8-22.

Ghosh, P., Satyawadi, R., Joshi, J.P., Rnjan, R., \& Singh, P. (2012). Towards more effective training programmes: a study of trainer attributes. Industrial and commercial training, 44(4), 194-202.

Gruber, H., Harteis, C., Kraft, S. (2004). Aufgaben- und Tätigkeitsfelder von Weiterbildner/inne/n. Vortrag auf der DIE-Veranstaltung ,Situation des Weiterbildungspersonals“ in Bonn am 19. November 2004. Unveröffentlichtes Manuskript (erhältlich über kraft@die-bonn.de).

Harris, T. B., Chung, W., Hutchins, H. M., \& Chiaburu, D. S. (2014a). Do trainer style and learner orientation predict training outcomes? Journal of Workplace Learning, 26(5), 331-344.

Harris, T. B., Chung, W., Frye, C. L., \& Chiaburu, D. S. (2014b). Satisfaction guaranteed? Enhanced impact of trainer competence for autonomous trainees. Industrial and commercial training, 46(5), 270-277.

Hippel, A. von, \& Fuchs, S. (2009). Aufgaben- und Tätigkeitsprofile von Weiterbildner/innen. In A. von Hippel \& R. Tippelt (Hrsg.). Fortbildung der Weiterbildner/innen. Eine Analyse der Interessen und Bedarfe aus verschiedenen Perspektiven (S. 63-88). Weinheim, Basel: Beltz.

Hohenstein, F., Zimmermann, F., Kleickmann, T., Köller, O., \& Möller, J. (2014). Sind die bildungswissenschaftlichen Standards für die Lehramtsausbildung in den Curricula der Hochschulen angekommen? Zeitschrift für Erziehungswissenschaft, 17(3), 497-507.

Holsti, O. R. (1969). Content analysis for the social sciences and humanities. Reading: Addison-Wesley.

Hutchins, H. M. (2009). In the trainer's voice: A study of training transfer practices. Performance Improvement Quarterly, 22(1), 69-93.

Hutchins, H. M., \& Burke, L. A. (2007). Identifying trainers' knowledge of training transfer research findings - closing the gap between research and practice. International Journal of Training and. Development, 11(4), 236-264.

Khamarko, K., Koester, K. A., Bie, J., Baron, R. B., \& Myers, J. J. (2012). Developing Effective Clinical Trainers: Strategies to Enhance Knowledge Translation. SAGE Open, 2(2). doi:10.1177/2158244012448486.

Kraft, S. (2006). Aufgaben und Tätigkeiten von Weiterbildner/inne/n - Herausforderungen und Perspektiven einer weiteren Professionalisierung in der Weiterbildung. http://www.die-bonn.de/esprid/ dokumente/doc-2006/kraft06_02.pdf. Zugegriffen: 10. Mai 2016.

Kraft, S., Seitter, W., \& Kollewe, L. M. (2009). Professionalitätsentwicklung des Weiterbildungspersonals. Bielefeld: W. Bertelsmann.

Kraft, S. (2011). Berufsfeld Weiterbildung. In R. Tippelt, \& A. von Hippel (Hrsg.), Handbuch Erwachsenenbildung/Weiterbildung (5. Aufl. S. 405-426). Wiesbaden: Springer VS. 
Kraft, S. (2013). Professionalitätsentwicklung in der Weiterbildung - Quo vadis? In T. C. Feld, S. Kraft, S. May, \& W. Seitter (Hrsg.). Engagierte Beweglichkeit. Weiterbildung in öffentlicher Verantwortung. Festschrift für Klaus Meisel (S. 245-256). Wiesbaden: Springer VS.

Krattenmacher, S., Brühwiler, C., Oser, F., \& Biedermann, H. (2010). Was angehende Lehrpersonen in den Erziehungswissenschaften lernen sollen. Curriculumanalyse der erziehungswissenschaftlichen Ausbildung an den Deutschschweizer Lehrerbildungsinstitutionen. Schweizerische Zeitschrift für Bildungswissenschaften, 32(1), 59-86.

Krüger, H.-H., Rauschenbach, T., Fuchs, K., Grunerst, C., Huber, A., Kleifgen, B., Rostampour, P., Seeling, C., \& Züchner, I. (2003). Diplom-Pädagogen in Deutschland: Survey 2001. Weinheim: Juventa.

Lenk, C. (2010). Freiberufler in der Weiterbildung: Empirische Studie am Beispiel Hessen. Erwachsenenbildung und lebensbegleitendes Lernen. Bd. 16. Bielefeld: W. Bertelsmann.

Lohmann, V., Seidel, V., \& Terhart, E. (2011). Bildungswissenschaften in der universitären Lehrerbildung: Curriculare Strukturen und Verbindlichkeiten. Lehrerbildung auf dem Prüfstand, 4(2), 271-302.

Mania, E., \& Strauch, A. (2010). Personal in der Weiterbildung. In Deutsches Institut für Erwachsenenbildung (Hrsg.), DIE spezial. Trends der Weiterbildung. DIE-Trendanalyse 2010 (S. 75-92). Bielefeld: W. Bertelsmann.

Marx, C., Goeze, A., \& Schrader, J. (2014a). Adult education teachers' pedagogical-psychological knowledge. Potential elements and test development. In S. Lattke \& W. Jütte (Hrsg.). Professionalisation of Adult Educators: International and Comparative Perspectives (pp. 165-182). Frankfurt a. M.: Lang.

Marx, C., Goeze, A., \& Schrader, J. (2014b). Pädagogisch-psychologisches Wissen zur Gestaltung von Lehr-Lernsituationen: (Wie) Unterscheidet es sich in Erwachsenenbildung/Weiterbildung und Schule? Hessische Blätter für Volksbildung, 3, 238-251.

Mayring, P. (2010). Qualitative Inhaltsanalyse: Grundlagen und Techniken (11. Aufl.). Weinheim: Beltz.

Nittel, D., Schütz, J., \& Tippelt, R. (2014). Pädagogische Arbeit im System des lebenslangen Lernens: Ergebnisse komparativer Berufsgruppenforschung. Weinheim, Basel: Beltz Juventa.

Rasli, A., Tat, H.H., Chin, T. A., \& Khalaf, B. (2012). Identification of factors and attributes for effective transfer of IT training to the workplace. Procedia - Social and Behavioral. Sciences, 40, 174-181.

Russ-Eft, D.F., Dickison, P., \& Levine, R. (2005). Instructor quality affecting emergency medical technician (EMT) preparedness: A LEADS Project. International Journal of Training and. Development, 9(4), 256-270.

Salas, E., Tannenbaum, S.I., Kraiger, K., \& Smith-Jentsch, K. A. (2012). The Science of Training and Development in Organizations: What matters in practice. Psychological Science in the Public Interest, 13(2), 74-101.

Schrader, J. (2013). Förderung der Kompetenzen von Lehrkräften, Trainern und Beratern durch die Arbeit mit Videofällen: Grundlagen und Strategien eines längerfristig angelegten Forschungs- und Entwicklungsprogramms. In S. Digel \& J. Schrader (Hrsg.). Diagnostizieren und Handeln von Lehrkräften. Lernen aus Videofällen in Hochschule und Erwachsenenbildung (S. 7-23). Bielefeld: W. Bertelsmann.

Schüßler, I. (2012). Studierenden- und Absolvent/inn/enbefragungen unter professionstheoretischer Perspektive. In R. Egetenmeyer, \& I. Schüßler (Hrsg.), Akademische Professionalisierung in der Erwachsenenbildung/Weiterbildung (S. 109-147). Baltmannsweiler: Schneider.

Schütz, J., \& Nittel, D. (2012). Von der Heterogenität zur Vielfalt. Akademische Professionalisierung im Blick einer komparativen pädagogischen Berufsgruppenforschung. In R. Egetenmeyer, \& D. Nittel (Hrsg.), Akademische Professionalisierung in der Erwachsenenbildung/Weiterbildung Grundlagen der Berufs- und Erwachsenenbildung, (Bd. 70, S. 229-244). Baltmannsweiler: Schneider.

Seyda, S., \& Werner, D. (2014). IW-Weiterbildungserhebung 2014 - Höheres Engagement und mehr Investitionen in betriebliche Weiterbildung. IW-Trends - Vierteljahresschrift zur empirischen Wirtschaftsforschung, 41(4), 1-15.

Sitzmann, T., Brown, K. G., Casper, W. J., Ely, K., \& Zimmerman, R. D. (2008). A review and meta-analysis of the nomological network of trainee reactions. Journal of Applied Psychology, 93(2), 280-295.

Stisser, A., Horn, K.-P., Züchner, I., Ruberg, C., \& Wigger, L. (2012). Studiengänge und Standorte. In W. Thole, H. Faulstich-Wieland, K.-P. Horn, H. Weishaupt, \& I. Züchner (Hrsg.). Datenreport Erziehungswissenschaft 2012. Schriften der deutschen Gesellschaft für Erziehungswissenschaft (S. 17-59). Opladen: Budrich.

Terry, J. (2010). Experiences of instructors delivering the Mental Health First Aid training programme: a descriptive qualitative study. Journal of Psychiatric and Mental Health Nursing, 17(7), 594-602.

Thole, W., Faulstich-Wieland, H., Horn, K.-P., Weishaupt, H., \& Züchner, I. (Hrsg.). (2012). Datenreport Erziehungswissenschaft 2012. Opladen: Budrich. 
Towler, A.J., \& Dipboye, R.L. (2001). Effects of trainer expressiveness, organization, and trainee goal orientation on training outcomes. Journal of Applied Psychology, 86(4), 664-673.

Voss, T., Kunina-Habenicht, O., Hoehne, V., \& Kunter, M. (2015). Stichwort Pädagogisches Wissen von Lehrkräften: Empirische Zugänge und Befunde. Zeitschrift für Erziehungswissenschaft, 18(2), 187-223.

Wißhak, S., \& Hochholdinger, S. (2015). „Zaubern” lernen - Welche pädagogischen Inhalte umfassen sogenannte Trainerausbildungen? Zeitschrift für Weiterbildungsforschung - Report, 38(1), 113-127.

WSF - Wirtschafts- und Sozialforschung (2005). Erhebung zur beruflichen und sozialen Lage von Lehrenden in Weiterbildungseinrichtungen: Schlussbericht. Kerpen. 